\title{
STRUCTURAL RESOLVENT ESTIMATES AND DERIVATIVE NONLINEAR SCHRÖDINGER EQUATIONS
}

\author{
MICHAEL RUZHANSKY AND MITSURU SUGIMOTO
}

\begin{abstract}
A refinement of uniform resolvent estimate is given and several smoothing estimates for Schrödinger equations in the critical case are induced from it. The relation between this resolvent estimate and radiation condition is discussed. As an application of critical smoothing estimates, we show a global existence results for derivative nonlinear Schrödinger equations.
\end{abstract}

\section{INTRODUCTION}

Let us consider the resolvent operator

$$
R(z)=(-\Delta-z)^{-1}
$$

on $\mathbb{R}^{n}$. It is defined only for $z \in \mathbb{C} \backslash\{x \in \mathbb{R} ; x>0\}$ as an element of $\mathcal{L}\left(L^{2}, L^{2}\right)$, but the weak limit

$$
R(\lambda \pm i 0)=\lim _{\varepsilon \searrow 0} R(\lambda \pm i \varepsilon)
$$

exists in $\mathcal{L}\left(L_{k}^{2}, L_{-k}^{2}\right)$ for $k>1 / 2$, where $L_{m}^{2}(m \in \mathbb{R})$ denotes the set of functions $g$ such that the norm

$$
\|g\|_{L_{m}^{2}}=\left(\int_{\mathbb{R}^{n}}\left|\langle x\rangle^{m} g(x)\right|^{2} d x\right)^{1 / 2} ; \quad\langle x\rangle=\left(1+|x|^{2}\right)^{1 / 2}
$$

is finite. This fact was first pointed out by Agmon [A], and is called the limiting absorption principle. This principle can be justified by the resolvent estimate

$$
\sup _{\varepsilon>0}\|R(\lambda \pm i \varepsilon) v\|_{L_{-k}^{2}} \leq C_{\lambda}\|v\|_{L_{k}^{2}}
$$

for $\lambda>0$ and $k>1 / 2$. More generally, we have

$$
\left\|D^{\alpha} R(\lambda \pm i 0) v\right\|_{L_{-k}^{2}} \leq C_{\lambda}\|v\|_{L_{k}^{2}}
$$

for $\lambda \neq 0,|\alpha| \leq 2$, and $k>1 / 2$.

Furthermore, if $n \geq 2$, we have the uniform resolvent estimates

$$
\sup _{\lambda \in \mathbb{R}}\||D| R(\lambda \pm i 0) v\|_{L_{-k}^{2}} \leq C\|v\|_{L_{k}^{2}}
$$

or equivalently

$$
\sup _{\lambda \in \mathbb{R}}\left\|\sigma(X, D) R(\lambda \pm i 0) \sigma(X, D)^{*} v\right\|_{L^{2}} \leq C\|v\|_{L^{2}}
$$

for $\sigma(x, \xi)=\langle x\rangle^{-k}|\xi|^{1 / 2}$, where $k>1 / 2$. The uniform resolvent estimates such as (1.2) and (1.3) are used to show smoothing estimates for Schrödinger equations. See,

Date: November 18, 2018.

The first author was supported by the EPSRC Leadership Fellowship EP/G007233/1. 
for example, [KY], [W], [Ho1], [Ho2], [Su1], [Su2], [SuT], RS2]. We remark that we have used here the notation

$$
\sigma(X, D, Y)=(2 \pi)^{-n} \int_{\mathbb{R}^{n}} \int_{\mathbb{R}^{n}} e^{i(x-y) \cdot \xi} \sigma(x, y, \xi) u(y) d y d \xi
$$

as a pseudo-differential operator following Kumano-go $[\mathrm{Ku}]$. We usually abbreviate $X$ (resp. $Y)$ if $\sigma(x, y, \xi)$ does not depend on $x$ (resp. $y$ ). For $\sigma(X, D)$, we set $\sigma(X, D)^{*}=$ $\bar{\sigma}(Y, D)$.

The objective of this paper is to establish the following:

- In uniform resolvent estimates (1.2) and (1.3), the critical case $k=1 / 2$ or more general combination of the order for the weight can be attained if we assume a structure on $\sigma(X, D)$ (Section 3 ).

- The structure is related to a radiation condition (Section 4).

- Such consideration can be used for the nonlinear problems for Schrödinger equations (Section 6).

Below we give the details, together with the organisation of this article. In Section 2. by following the argument in authors' previous work [RS2], we will show a refined version of the resolvent estimate (1.1), which is associated with a structure induced by $-\Delta$. To understand the geometric meaning of this structure, we will also consider more general elliptic operators $L$ instead of $-\triangle$. After such preliminary results, we will show in Section 3 a uniform resolvent estimate (Theorem 3.1) which includes the following result as a special case

Theorem 1.1. Let $n \geq 2$ and let $\theta \in \mathbb{R}$. Suppose that $\sigma(x, \xi) \sim|\xi|^{\theta}, \tau(x, \xi) \sim$ $|\xi|^{1-\theta}$, and $\sigma(x, \xi)=0$ on $\Gamma=\left\{(x, \xi) \in \mathbb{R}^{n} \times\left(\mathbb{R}^{n} \backslash 0\right) ; x \wedge \xi=0\right\}$. Then we have the estimate

$$
\sup _{\lambda \in \mathbb{R}}\left\|\sigma(X, D) R(\lambda \pm i 0) \tau(X, D)^{*} v\right\|_{L_{-1 / 2+l}^{2}\left(\mathbb{R}^{n}\right)} \leq C\|v\|_{L_{1 / 2+l}^{2}\left(\mathbb{R}^{n}\right)}
$$

$0<l<\min \{1,(n-1) / 2\}$. Suppose also that $\tau(x, \xi)=0$ on $\Gamma$. Then estimate (3.2) is true for $|l|<\min \{1,(n-1) / 2\}$.

Here the notation $\sigma(x, \xi) \sim|\xi|^{b}$ means that $\sigma(x, \xi)$ is positively homogeneous of order $b$ in the variable $\xi$ and its derivatives satisfy a natural decaying property. For the precise definition, see (3.1). Here we have also used the notation $a \wedge b=$ $\left(a_{i} b_{j}-a_{j} b_{i}\right)_{i<j}$ for vectors $a=\left(a_{1}, a_{2}, \ldots, a_{n}\right)$ and $b=\left(b_{1}, b_{2}, \ldots, b_{n}\right)$. Theorem 1.1 with $l=0$ corresponds to estimates (1.2) and (1.3) with the critical case $k=1 / 2$, and furthermore, we have freedom in the choice of $l$. Such advantages come from the extra structure conditions $\sigma(x, \xi)=0, \tau(x, \xi)=0$ on the set $\Gamma$. We remark that the case of homogeneous weight $|x|^{-1 / 2}$ instead of $\langle x\rangle^{-1 / 2}$ was considered in [RS2] (when $l=0)$.

In Section 4, we will explain the relation between our result Theorem 1.1 and Herbst-Skibsted's resolvent estimate in [HS], where the symbol $\sigma(x, \xi)=\xi \mp(x /|x|)|\xi|$ vanishing only on the half part of $\Gamma$ is used to show results similar to Theorem 1.1. We remark that such result induces Sommerfeld's radiation condition.

Theorem 1.1 implies many estimates for Schrödinger equations. For example, if we follow the terminology in [KY], Theorem 1.1 with the case $\sigma(x, \xi)=\tau(x, \xi)$ 
and $l=0$ means that the operator $\tilde{\sigma}(X, D)=\langle X\rangle^{-1 / 2} \sigma(X, D)$ is $-\Delta$-supersmooth. Due to Kato's work [K], this property automatically induces smoothing estimates for Schrödinger equations, which covers the critical case of the estimates obtained by Ben-Artzi and Klainerman [BK] or Chihara [Ch2]. Such results will be displayed in Section 5 .

As an application of smoothing estimates induced from Theorem 1.1, we will consider in Section 6 the existence of time global solutions to the following derivative nonlinear Schrödinger equation

$$
\left\{\begin{array}{c}
\left(i \partial_{t}+\triangle\right) u(t, x)=f(\nabla u(t, x)) \\
u(0, x)=\varphi(x), \quad t \in \mathbb{R}, x \in \mathbb{R}^{n},
\end{array}\right.
$$

where $\triangle$ and $\nabla$ denote the Laplacian and the gradient in $x \in \mathbb{R}^{n}$, respectively. The question is which conditions on the initial data $\varphi$ guarantee the global in time existence of solutions? Some answers in the case when $f(u)$ has a polynomial growth of order $N$ are known:

- $\varphi \in C^{\infty}$, rapidly decay, and sufficiently small in the case $N \geq 3$ (Chihara $[\mathrm{Ch}]$.

- $\varphi \in H^{[n / 2]+5}$, rapidly decay, and sufficiently small in the case $N \geq 2$ (Hayashi, Miao, and Naumkin [HMN]).

- $\varphi \in H^{n / 2+2}$, sufficiently small in the case $N \geq 3$ (Ozawa and Zhai [OZ]).

By using smoothing estimates obtained in Section 5 , we can weaken the smoothness assumption on $\varphi$ if the nonlinear term has a null-form structure like $f(x /\langle x\rangle \wedge \nabla u)$. As it was announced in [RS3], the regularity index $[n / 2]+5$, or even $n / 2+2$, can be replaced by a smaller one in this case. We give an example of these results:

Theorem 1.2. Let $n \geq 3$, let $N \geq 4, N \in \mathbb{N}$, let $\kappa>3(N+1) / 4(N-1)$, and let $\tau>(n+3) / 2$. Assume that $\langle x\rangle^{\kappa}\langle D\rangle^{\tau} \varphi \in L^{2}$ and its $L^{2}$-norm is sufficiently small. Then the equation

$$
\left\{\begin{array}{c}
\left(i \partial_{t}+\triangle\right) u(t, x)=|(x /\langle x\rangle) \wedge \nabla u|^{N}, \\
u(0, x)=\varphi(x), \quad t \in \mathbb{R}, x \in \mathbb{R}^{n}
\end{array}\right.
$$

has a time global solution $u \in C^{0}\left(\mathbb{R}_{t} \times \mathbb{R}_{x}^{n}\right)$.

The proof of Theorem 1.2 is a simple application of the fixed point theorem on contraction mapping. The freedom of the choice of $l$ in Theorem 1.1, which is due to the structure of nonlinear term, enables us to induce the contraction directly.

The phenomena that some structure of the nonlinear term has effects on the regularity problem can be seen in many nonlinear equations. For example, KlainermanMachedon KM1, KM2 showed that wave equations with nonlinear terms satisfying the null condition have local existence and uniqueness in the Sobolev space $H^{s}$ for smaller $s$ than that of wave equations with general nonlinear terms. Theorem 1.2 can be regarded as one of such phenomena for Schrödinger equations. 


\section{A Resolvent estimate With structure}

In this section, we establish a refined version of the resolvent estimate (1.3) in Introduction, which is associated with a structure induced by $-\Delta$. To describe it, we also generalise the operator $L=-\triangle$, which will also enable us to clarify its geometric meaning. For the purpose, we introduce notations which will be used in the rest of this paper. Let

$$
\begin{aligned}
L=p(D)^{2} ; \quad & p(\xi) \in C^{\infty}\left(\mathbb{R}^{n} \backslash 0\right), \quad p(\xi)>0, \quad p(\lambda \xi)=\lambda p(\xi) \quad(\lambda>0), \\
& \left\{\xi \in \mathbb{R}^{n} \backslash 0: p(\xi)=1\right\} \text { has non-vanishing Gaussian curvature. }
\end{aligned}
$$

Then $\psi(\xi)=p(\xi) \frac{\nabla p(\xi)}{|\nabla p(\xi)|}$ defines a $C^{\infty}$-diffeomorphism on $\mathbb{R}^{n} \backslash 0$, and we set

$$
\begin{aligned}
& \Omega(x, \xi)=x \psi^{\prime}(\xi)^{-1} \wedge \psi(\xi)=\left(\Omega_{i j}(x, \xi)\right)_{i<j}, \\
& \omega(x, \xi)=\langle x\rangle^{-1} \Omega(x, \xi)=\left(\omega_{i j}(x, \xi)\right)_{i<j} .
\end{aligned}
$$

We remark that $L=-\triangle$ and $\omega(x, \xi)=(x /\langle x\rangle) \wedge \xi$ if $p(\xi)=|\xi|$. We also set

$$
\begin{aligned}
& R(z)=(L-z)^{-1}=\mathcal{F}_{\xi}^{-1}\left(p(\xi)^{2}-z\right)^{-1} \mathcal{F}_{x}, \\
& R(\lambda \pm i 0)=\lim _{\varepsilon \searrow 0} R(\lambda \pm i \varepsilon)
\end{aligned}
$$

for $z \in \mathbb{C} \backslash\{x \in \mathbb{R} ; x \geq 0\}$ and $\lambda \in \mathbb{R}$, where $\mathcal{F}_{x}, \mathcal{F}_{\xi}^{-1}$ denote the Fourier transformation and its inverse, respectively, and the limit is taken in the sense of distributions. For $\lambda<0$, we have $R(\lambda \pm i 0)=R(\lambda)$. We remark that $\Omega$ commutes with functions of the operator $p(D)$.

Lemma 2.1. Let $h \in C^{\infty}(\mathbb{R} \backslash 0)$. Then the operators of the form $(h \circ p)(D)$ commute with $\Omega_{i j}(X, D)$ whenever it makes sense. In particular, $R(\lambda \pm i 0)$ commutes with $\Omega_{i j}(X, D)$.

Proof. See the proof of [RS2, Lemma 3.2].

Let us also introduce the classical orbit $\{(x(t), \xi(t)): t \in \mathbb{R}\}$ associated to the operator $L$ defined by (2.1), which satisfies

$$
\left\{\begin{array}{l}
\dot{x}(t)=\nabla_{\xi} p^{2}(\xi(t)), \quad \dot{\xi}(t)=0, \\
x(0)=0, \quad \xi(0)=\eta
\end{array}\right.
$$

and define the set of the paths of all classical orbits:

$$
\begin{aligned}
\Gamma & =\left\{(x(t), \xi(t)): t \in \mathbb{R}, \eta \in \mathbb{R}^{n} \backslash 0\right\} \\
& =\left\{(\lambda \nabla p(\xi), \xi): \xi \in \mathbb{R}^{n} \backslash 0, \lambda \in \mathbb{R}\right\} .
\end{aligned}
$$

In the case $L=-\triangle$, for example, we have

$$
\begin{aligned}
\Gamma & =\left\{(\lambda \xi, \xi): \xi \in \mathbb{R}^{n} \backslash 0, \lambda \in \mathbb{R}\right\} \\
& =\left\{(x, \xi) \in \mathbb{R}^{n} \times\left(\mathbb{R}^{n} \backslash 0\right): x \wedge \xi=0\right\} .
\end{aligned}
$$

By using it, we define a structure induced by the operator $L$ :

$$
\sigma(x, \xi)=0 \quad \text { if } \quad(x, \xi) \in \Gamma .
$$


We remark that $\omega_{i j}(x, \xi)$ in (2.2) satisfy (2.5) (see [RS2, Lemma 3.1]). With the dual function $p^{*}(x)$ of $p(x)$ which satisfies $p^{*}(\nabla p(x))=1$ (see [RS2, Theorem 3.1]), elements of

$$
\omega^{*}(x, \xi)=a(x) \nabla p^{*}(x) \wedge \xi
$$

are also examples of $\sigma(x, \xi)$ which satisfies (2.5), where $a(x)$ is an appropriate function whose support is away from the origin.

We say that $\sigma(x, \xi)$ is of the class $\mathcal{A}_{k}^{m}$ if it satisfies

$$
\left|\partial_{x}^{\alpha} \partial_{\xi}^{\gamma} \sigma(x, \xi)\right| \leq C_{\alpha \gamma}\langle x\rangle^{m-|\alpha|}\langle\xi\rangle^{k-|\gamma|}
$$

for all $\alpha$ and $\gamma$. In the case $k=0$, we abbreviate it writing $\mathcal{A}^{m}$. Then we have the following proposition:

Proposition 2.2. Let $n \geq 2$, let $\lambda \in \mathbb{R}$, and let $\chi \in C_{0}^{\infty}\left(\mathbb{R}^{n} \backslash 0\right)$. Suppose that $\sigma(x, \xi) \in \mathcal{A}^{-1 / 2+l}, \tau(x, \xi) \in \mathcal{A}^{-1 / 2-l}$ and $\sigma(x, \xi)=0$ on $\Gamma$. Then there is some $N \in \mathbb{N}$ such that we have the estimate

$$
\begin{aligned}
& \left\|\sigma(X, D) R(\lambda \pm i 0) \chi(D) \tau(X, D)^{*} v\right\|_{L^{2}\left(\mathbb{R}^{n}\right)} \\
\leq & C_{\lambda, \chi}\left(\sup _{\substack{x, \xi \in \mathbb{R}^{n} \\
|\alpha|+|\gamma| \leq N}}\left|\frac{\partial_{x}^{\alpha} \partial_{\xi}^{\gamma} \sigma(x, \xi)}{\langle x\rangle^{-1 / 2+l-|\alpha|}\langle\xi\rangle^{-|\gamma|}}\right|\right)\left(\sup _{\substack{x, \xi \in \mathbb{R}^{n} \\
|\alpha|+|\gamma| \leq N}}\left|\frac{\partial_{x}^{\alpha} \partial_{\xi}^{\gamma} \tau(x, \xi)}{\langle x\rangle^{-1 / 2-l-|\alpha|}\langle\xi\rangle^{-|\gamma|}}\right|\right)\|v\|_{L^{2}\left(\mathbb{R}^{n}\right)}
\end{aligned}
$$

for $0<l<1$. Suppose also that $\tau(x, \xi)=0$ on $\Gamma$. Then the estimate is true for $-1<l<1$.

We will now prove Proposition 2.2. The proof is a modified version of the arguments in [Su2, Theorem 3.1] and [RS2, Theorem 4.1] and it may include the repetition of them. The following lemma due to [RS2, Proposition 3.3] (and its proof) is essential:

Lemma 2.3. Let $m \in \mathbb{R}$, let $\varepsilon>0$, and let $\sigma(x, \xi) \in \mathcal{A}_{1}^{m}$. Suppose that $\sigma(x, \xi)=0$ if $(x, \xi) \in \Gamma$ or $|\xi|<\varepsilon$. Then we have

$$
\begin{aligned}
& \|\sigma(X, D) u\|_{L^{2}\left(\mathbb{R}^{n}\right)} \\
\leq & C\left(\sup _{\substack{x, \xi \in \mathbb{R}^{n} \\
|\alpha|+|\gamma| \leq N}}\left|\frac{\partial_{x}^{\alpha} \partial_{\xi}^{\gamma} \sigma(x, \xi)}{\langle x\rangle^{m-|\alpha|}\langle\xi\rangle^{1-|\gamma|}}\right|\right)\left(\sum_{i<j}\left\|\Omega_{i j}(X, D) u\right\|_{L_{m-1}^{2}\left(\mathbb{R}^{n}\right)}+\|u\|_{L_{m-1}^{2}\left(\mathbb{R}^{n}\right)}\right),
\end{aligned}
$$

with a sufficiently large $N \in \mathbb{N}$.

We set

$$
K_{\lambda, \chi}=R(\lambda \pm i 0) \chi(D) .
$$

By Lemma 2.3 and by taking the adjoint, the estimate of Proposition 2.2 is reduced to show the $L^{2}\left(\mathbb{R}^{n}\right)$-boundedness of the operator

$$
\widetilde{K}_{\lambda, \chi}=\langle x\rangle^{-3 / 2+l}\left(\Omega_{i j}\right)^{k} K_{\lambda, \chi}\langle x\rangle^{-1 / 2-l},
$$

for $0<l<1$, and

$$
\widetilde{K}_{\lambda, \chi}=\langle x\rangle^{-3 / 2+l}\left(\Omega_{i j}\right)^{k} K_{\lambda, \chi}\left(\Omega_{i^{\prime} j^{\prime}}^{*}\right)^{k^{\prime}}\langle x\rangle^{-3 / 2-l}
$$


for $-1<l<1$, where $\Omega_{i^{\prime} j^{\prime}}^{*}$ is the adjoint of $\Omega_{i^{\prime} j^{\prime}}$, and $k, k^{\prime}=0,1$.

Here we have also used the boundedness of $\tau(X, D)$ from $L_{-1 / 2-l}^{2}$ to $L^{2}$, which is justified by [RS1, Theorem 3.1], [RS2, Theorem 2.1]. In any case, since $\Omega_{i j}$ almost commutes with $K_{\lambda, \chi}$ by Lemma 2.1, $\widetilde{K}_{\lambda, \chi}$ has the expressions

$$
\begin{aligned}
\widetilde{K}_{\lambda, \chi} & =\sum_{\nu: \text { finite }}\langle x\rangle^{-3 / 2+l} R(\lambda \pm i 0) \chi_{\nu}(D) f_{\nu}(x)\langle x\rangle^{-3 / 2-l} \\
& =\sum_{\nu: \text { finite }}\langle x\rangle^{-3 / 2+l} \tilde{f}_{\nu}(x) R(\lambda \pm i 0) \tilde{\chi}_{\nu}(D)\langle x\rangle^{-3 / 2-l}
\end{aligned}
$$

where $f_{\nu}, \tilde{f}_{\nu}$ are functions of polynomial growth of order 2 at most, and $\chi_{\nu}, \tilde{\chi}_{\nu} \in C_{0}^{\infty}$ have their support in that of $\chi$. Hence we may assume

$$
\widetilde{K}_{\lambda, \chi}=\langle x\rangle^{-3 / 2+l} K_{\lambda, \chi}\langle x\rangle^{1 / 2-l}, \quad \widetilde{K}_{\lambda, \chi}=\langle x\rangle^{1 / 2+l} K_{\lambda, \chi}\langle x\rangle^{-3 / 2-l},
$$

whichever we need to show the $L^{2}$ boundedness for $-1<l<1$.

We may assume, as well, that $\chi(\xi) \in C_{0}^{\infty}\left(\mathbb{R}^{n}\right)$ has its support in a sufficiently small conic neighbourhood of $(0, \ldots, 0,1)$. We split the variables in $\mathbb{R}^{n}$ in the way of

$$
x=\left(x^{\prime}, x_{n}\right), x^{\prime}=\left(x_{1}, \ldots, x_{n-1}\right) .
$$

By the integral kernel representation, we express the operators $\widetilde{K}_{\lambda, \chi}$ and $K_{\lambda, \chi}$ as

$$
\begin{aligned}
\widetilde{K}_{\lambda, \chi} v(x) & =\int \widetilde{K}_{\lambda, \chi}(x, y) v(y) d y=\int d y_{n} \cdot \int \widetilde{K}_{\lambda, \chi}(x, y) v(y) d y^{\prime}, \\
K_{\lambda, \chi} v(x) & =\int K_{\lambda, \chi}(x, y) v(y) d y=\int d y_{n} \cdot \int K_{\lambda, \chi}(x, y) v(y) d y^{\prime} .
\end{aligned}
$$

The following is fundamental in the proof of the limiting absorption principle (see [RS2, Lemma 4.1]):

Lemma 2.4. Let $\chi(\xi) \in C_{0}^{\infty}\left(\mathbb{R}^{n}\right)$ have its support in a small conic neighbourhood of $(0, \ldots, 0,1)$. Then we have

$$
\left\|\int K_{\lambda, \chi}(x, y) v(y) d y^{\prime}\right\|_{L^{2}\left(\mathbb{R}_{x^{\prime}}^{n-1}\right)} \leq C_{\lambda, \chi}\left\|v\left(\cdot, y_{n}\right)\right\|_{L^{2}\left(\mathbb{R}^{n-1}\right)},
$$

where $C_{\lambda, \chi}$ is independent of $x_{n}$ and $y_{n}$.

By (2.6) and Lemma 2.4, we have easily

$$
\left\|\int\langle x\rangle^{3 / 2-l} \widetilde{K}_{\lambda, \chi}(x, y)\langle y\rangle^{-1 / 2+l} v(y) d y^{\prime}\right\|_{L^{2}\left(\mathbb{R}_{x^{\prime}}^{n-1}\right)} \leq C_{\lambda, \chi}\left\|v\left(\cdot, y_{n}\right)\right\|_{L^{2}\left(\mathbb{R}^{n-1}\right)}
$$

and

$$
\left\|\int\langle x\rangle^{-1 / 2-l} \widetilde{K}_{\lambda, \chi}(x, y)\langle y\rangle^{3 / 2+l} v(y) d y^{\prime}\right\|_{L^{2}\left(\mathbb{R}_{x^{\prime}}^{n-1}\right)} \leq C_{\lambda, \chi}\left\|v\left(\cdot, y_{n}\right)\right\|_{L^{2}\left(\mathbb{R}^{n-1}\right)} .
$$

By interpolation, we have

$$
\left\|\int\langle x\rangle^{1 / 2 \pm \epsilon} \widetilde{K}_{\lambda, \chi}(x, y)\langle y\rangle^{1 / 2 \mp \epsilon} v(y) d y^{\prime}\right\|_{L^{2}\left(\mathbb{R}_{x^{\prime}}^{n-1}\right)} \leq C_{\lambda, \chi}\left\|v\left(\cdot, y_{n}\right)\right\|_{L^{2}\left(\mathbb{R}^{n-1}\right)},
$$


hence

$$
\left\|\int \widetilde{K}_{\lambda, \chi}(x, y) v(y) d y^{\prime}\right\|_{L^{2}\left(\mathbb{R}_{x^{\prime}}^{n-1}\right)} \leq C_{\lambda, \chi} \frac{\left\|v\left(\cdot, y_{n}\right)\right\|_{L^{2}\left(\mathbb{R}^{n-1}\right)}}{\left|x_{n}\right|^{1 / 2 \pm \epsilon \epsilon}\left|y_{n}\right|^{1 / 2 \mp \epsilon}}
$$

for $0<\epsilon \leq 1-|l|$. Since $\left|x_{n}\right|^{-2 \epsilon} \leq 2^{2 \epsilon}\left|x_{n}-y_{n}\right|^{-2 \epsilon}$ if $\left|x_{n}\right| \geq\left|y_{n}\right|$ and $\left|y_{n}\right|^{-2 \epsilon} \leq$ $2^{2 \epsilon}\left|x_{n}-y_{n}\right|^{-2 \epsilon}$ if $\left|x_{n}\right| \leq\left|y_{n}\right|$, we have

$$
\left\|\int \widetilde{K}_{\lambda, \chi}(x, y) v(y) d y^{\prime}\right\|_{L^{2}\left(\mathbb{R}_{x^{\prime}}^{n-1}\right)} \leq C_{\lambda, \chi} \frac{\left\|v\left(\cdot, y_{n}\right)\right\|_{L^{2}\left(\mathbb{R}^{n-1}\right)}}{\left|x_{n}\right|^{1 / 2-\epsilon}\left|x_{n}-y_{n}\right|^{2 \epsilon}\left|y_{n}\right|^{1 / 2-\epsilon}} .
$$

If we take $\epsilon$ such that $0<\epsilon<\min \{1 / 2,1-|l|\}$, then we have

$$
\begin{aligned}
\left\|\widetilde{K}_{\lambda, \chi} v\right\|_{L^{2}\left(\mathbb{R}^{n}\right)} & \leq\left\|\int\right\| \int \widetilde{K}_{\lambda, \chi}(x, y) v(y) d y^{\prime}\left\|_{L^{2}\left(\mathbb{R}_{x^{\prime}}^{n-1}\right)} d y_{n}\right\|_{L^{2}\left(\mathbb{R}_{x_{n}}\right)} \\
& \leq C_{\lambda, \chi}\left\|\int \frac{\left\|v\left(\cdot, y_{n}\right)\right\|_{L^{2}\left(\mathbb{R}^{n-1}\right)}}{\left|x_{n}\right|^{1 / 2-\epsilon}\left|x_{n}-y_{n}\right|^{2 \epsilon}\left|y_{n}\right|^{1 / 2-\epsilon}} d y_{n}\right\|_{L^{2}\left(\mathbb{R}_{x_{n}}\right)} \\
& \leq C_{\lambda, \chi}\|v\|_{L^{2}\left(\mathbb{R}^{n}\right)},
\end{aligned}
$$

where we have used the following fact (with the case $n=1$ ) proved by HardyLittlewood [HL]:

Lemma 2.5. Let $\gamma<n / 2, \delta<n / 2, m<n$, and $\gamma+\delta+m=n$. Then we have

$$
\begin{aligned}
\left(\left.\left.\int_{\mathbb{R}^{n}}|| x\right|^{-\gamma}|D|^{m-n}|x|^{-\delta} f(x)\right|^{2} d x\right)^{1 / 2} & =\left(\int_{\mathbb{R}^{n}}\left|\int_{\mathbb{R}^{n}} \frac{f(y)}{|x|^{\gamma}|x-y|^{m}|y|^{\delta}} d y\right|^{2} d x\right)^{1 / 2} \\
& \leq C\left(\int_{\mathbb{R}^{n}}|f(x)|^{2} d x\right)^{1 / 2} .
\end{aligned}
$$

(See also [SW, Theorem B].) Thus we have completed the proof of Proposition 2.2.

\section{UNIFORM RESOLVENT ESTIMATES}

In this section we derive uniform resolvent estimates. We use the notation

$$
\sigma(x, \xi) \sim|\xi|^{b} \quad(b \in \mathbb{R})
$$

in the sense that it satisfies $\sigma(x, \xi) \in C^{\infty}\left(\mathbb{R}_{x}^{n} \times\left(\mathbb{R}_{\xi}^{n} \backslash 0\right)\right)$ and

$$
\sigma(x, \lambda \xi)=\lambda^{b} \sigma(x, \xi) ;(\lambda>0, \xi \neq 0), \quad\left|\partial_{x}^{\alpha} \sigma(x, \xi)\right| \leq C_{\alpha}\langle x\rangle^{-|\alpha|}|\xi|^{b} .
$$

If $b=1$, we write $\sigma(x, \xi) \sim|\xi|$. Then Proposition 2.2 given in Section 2 induces the following uniform resolvent estimate:

Theorem 3.1. Let $n \geq 2$ and let $\theta \in \mathbb{R}$. Suppose that $\sigma(x, \xi) \sim|\xi|^{\theta}, \tau(x, \xi) \sim|\xi|^{1-\theta}$, and $\sigma(x, \xi)=0$ on $\Gamma$. Then we have the estimate

$$
\sup _{\lambda \in \mathbb{R}}\left\|\sigma(X, D) R(\lambda \pm i 0) \tau(X, D)^{*} v\right\|_{L_{-1 / 2+l}^{2}\left(\mathbb{R}^{n}\right)} \leq C\|v\|_{L_{1 / 2+l}^{2}\left(\mathbb{R}^{n}\right)}
$$

$0<l<\min \{1,(n-1) / 2\}$. Suppose also that $\tau(x, \xi)=0$ on $\Gamma$. Then estimate (3.2) is true for $|l|<\min \{1,(n-1) / 2\}$. 
As a special case of Theorem 3.1, we have

Corollary 3.2. Let $n \geq 2$. Suppose that $\sigma(x, \xi) \sim|\xi|^{1 / 2}$ and $\sigma(x, \xi)=0$ on $\Gamma$. Then we have

$$
\sup _{\lambda \in \mathbb{R}}\left\|\sigma(X, D) R(\lambda \pm i 0) \sigma(X, D)^{*} v\right\|_{L_{-1 / 2}^{2}\left(\mathbb{R}^{n}\right)} \leq C\|v\|_{L_{1 / 2}^{2}\left(\mathbb{R}^{n}\right)}
$$

Suppose that $\sigma(x, \xi) \sim|\xi|$ and $\sigma(x, \xi)=0$ on $\Gamma$. Then we have

$$
\sup _{\lambda \in \mathbb{R}}\|\sigma(X, D) R(\lambda \pm i 0) v\|_{L_{-1 / 2+l}^{2}\left(\mathbb{R}^{n}\right)} \leq C\|v\|_{L_{1 / 2+l}^{2}\left(\mathbb{R}^{n}\right)}
$$

for $0<l<1$ if $n \geq 3$ and $0<l<1 / 2$ if $n=2$.

We now prove Theorem 3.1. We may consider only the case of non-negative $l$ since the estimate for negative $l$ is also given by the duality argument. That is, it suffices to show estimate (3.2) for $0<l<\min \{1,(n-1) / 2\}$ assuming that $\sigma(x, \xi)=0$ on $\Gamma$, and for $l=0$ assuming that $\sigma(x, \xi)=\tau(x, \xi)=0$ on $\Gamma$. We split estimate (3.2) into the following two estimates:

$$
\begin{gathered}
\sup _{\lambda \neq 0}\left\|\sigma(X, D) R(\lambda \pm i 0) \tau(X, D)^{*} v\right\|_{L_{-1 / 2+l}^{2}\left(\mathbb{R}^{n}\right)} \leq C\|v\|_{L_{1 / 2+l}^{2}\left(\mathbb{R}^{n}\right)}, \\
\left\|\sigma(X, D) R(0 \pm i 0) \tau(X, D)^{*} v\right\|_{L_{-1 / 2+l}^{2}\left(\mathbb{R}^{n}\right)} \leq C\|v\|_{L_{1 / 2+l}^{2}\left(\mathbb{R}^{n}\right)} .
\end{gathered}
$$

The proof of estimate $(3.6)$ is reduced to show the $L^{2}$-boundedness of the operator $A(X, D, Y)=\langle X\rangle^{-1 / 2+l} \sigma(X, D) p(D)^{-2} \tau(X, D)^{*}\langle Y\rangle^{-1 / 2-l}$. Since $\langle x\rangle^{-1 / 2+l} \leq$ $C\left(|x|^{-1 / 2}+|x|^{-1 / 2+l}\right)$ and $\langle x\rangle^{-1 / 2-l} \leq \min \left\{|x|^{-1 / 2},|x|^{-1 / 2-l}\right\}$, it is further reduced to that of $A(X, D, Y)=|X|^{-1 / 2+l} \sigma(X, D) p(D)^{-2} \tau(X, D)^{*}|Y|^{-1 / 2-l}$ with $0 \leq l<$ $(n-1) / 2$, which is obtained from the following lemma (with $b=1$ and $\delta=1 / 2-l$ ):

Lemma 3.3. Let $\delta<n / 2,0<b<\delta+n / 2$. Suppose that $A(x, \cdot, y) \in C^{\infty}\left(\mathbb{R}^{n} \backslash 0\right)$ and

Then we have

$$
A(x, \lambda \xi, y)=\lambda^{-b} A(x, \xi, y) \quad\left(\lambda>0, x, y \in \mathbb{R}^{n} \backslash 0\right)
$$

$$
\|A(X, D, Y) u\|_{L^{2}\left(\mathbb{R}^{n}\right)} \leq C\left(\left.\sup _{\substack{x, y \in \mathbb{R}^{n} \backslash 0 \\|\xi|=1,|\gamma| \leq 2 n}}|| x\right|^{\delta} \partial_{\xi}^{\gamma} A(x, \xi, y)|y|^{b-\delta} \mid\right)\|u\|_{L^{2}\left(\mathbb{R}^{n}\right)} .
$$

Proof. We have

where

$$
A(X, D, Y) u(x)=\int K(x, x-y, y) u(y) d y
$$

$$
K(x, z, y)=\mathcal{F}_{\xi}^{-1}[A(x, \xi, y)](z)
$$

Taking a cutoff function $\chi(\xi) \in C_{0}^{\infty}\left(\mathbb{R}^{n}\right)$ such that $\chi(\xi)=1$ near $\xi=0$, we have

$$
\begin{aligned}
K(x, z, y) & =(2 \pi)^{-n} \int e^{i \xi \cdot z} A(x, \xi, y) \chi(\xi) d \xi \\
& +(2 \pi)^{-n}|z|^{-2 n} \int e^{i \xi \cdot z}\left(-\triangle_{\xi}\right)^{n}(A(x, \xi, y)(1-\chi)(\xi)) d \xi
\end{aligned}
$$


by integration by parts. Then we have

$$
\sup _{|z|=1}|K(x, z, y)| \leq C \sup _{\substack{\xi \neq 0 \\|\gamma| \leq 2 n}}|\xi|^{b+|\gamma|}\left|\partial_{\xi}^{\gamma} A(x, \xi, y)\right|=C \sup _{\substack{|\xi|=1 \\|\gamma| \leq 2 n}}\left|\partial_{\xi}^{\gamma} A(x, \xi, y)\right|,
$$

hence

$$
|K(x, z, y)| \leq C \sup _{\substack{|\xi|=1 \\|\gamma| \leq 2 n}}\left|\partial_{\xi}^{\gamma} A(x, \xi, y)\right||z|^{-(n-b)}
$$

since $K(x, \lambda z, y)=\lambda^{b-n} K(x, z, y)$ for $\lambda>0$ and $x, y \in \mathbb{R}^{n}$. From this, we obtain

$$
|A(X, D, Y) u(x)| \leq C\left(\left.\sup _{\substack{x, y \in \mathbb{R}^{n}|0\\| \xi|=1,| \gamma \mid \leq 2 n}}|| x\right|^{\delta} \partial_{\xi}^{\gamma} A(x, \xi, y)|y|^{b-\delta} \mid\right) \int \frac{|u(y)|}{|x|^{\delta}|x-y|^{n-b}|y|^{b-\delta}} d y,
$$

which implies the result by Lemma 2.5 .

We show estimate (3.5) by the scaling argument. Noting that we have generally

$$
a(X, D, Y) f(x)=a\left(|\lambda|^{-1} X,|\lambda| D,|\lambda|^{-1} Y\right)\left[f\left(|\lambda|^{-1} \cdot\right)\right](|\lambda| x),
$$

estimate (3.5) is reduced to showing the estimates

$$
\begin{aligned}
& \sup _{\lambda>0}\left\|\sigma_{\lambda}(X, D) R(1 \pm i 0) \tau_{\lambda}(X, D)^{*} v\right\|_{L^{2}\left(\mathbb{R}^{n}\right)} \leq C\|v\|_{L^{2}\left(\mathbb{R}^{n}\right)}, \\
& \sup _{\lambda>0}\left\|\sigma_{\lambda}(X, D) R(-1 \pm i 0) \tau_{\lambda}(X, D)^{*} v\right\|_{L^{2}\left(\mathbb{R}^{n}\right)} \leq C\|v\|_{L^{2}\left(\mathbb{R}^{n}\right)},
\end{aligned}
$$

where we set

$$
\begin{aligned}
& \sigma_{\lambda}(x, \xi)=\lambda^{-1 / 2}\left\langle\lambda^{-1} x\right\rangle^{-1 / 2+l} \sigma\left(\lambda^{-1} x, \xi\right), \\
& \tau_{\lambda}(x, \xi)=\lambda^{-1 / 2}\left\langle\lambda^{-1} x\right\rangle^{-1 / 2-l} \tau\left(\lambda^{-1} x, \xi\right) .
\end{aligned}
$$

If $l>1 / 2$, for $x$ such that $\left|\lambda^{-1} x\right| \geq 2$, we have $\lambda^{-1 / 2}\left\langle\lambda^{-1} x\right\rangle^{-1 / 2+l} \leq C \lambda^{-l}|x|^{-1 / 2+l}$, and for $x$ such that $\left|\lambda^{-1} x\right| \leq 2$, we have $\lambda^{-1 / 2}\left\langle\lambda^{-1} x\right\rangle^{-1 / 2+l} \leq C \lambda^{-1 / 2}\left\langle\lambda^{-1} x\right\rangle^{-1 / 2+l^{\prime}} \leq$ $C \lambda^{-l^{\prime}}|x|^{-1 / 2+l^{\prime}}$ for $l^{\prime} \leq 1 / 2$. Hence we have

$$
\begin{aligned}
& \left\|\sigma_{\lambda}(X, D) R( \pm 1 \pm i 0) \tau_{\lambda}(X, D)^{*} v\right\|_{L^{2}\left(\mathbb{R}^{n}\right)} \\
\leq & \left(\left(\int_{\left|\lambda^{-1} x\right| \geq 2}+\int_{\left|\lambda^{-1} x\right| \leq 2}\right)\left|\sigma_{\lambda}(X, D) R( \pm 1 \pm i 0) \tau_{\lambda}(X, D)^{*} v\right|^{2} d x\right)^{1 / 2} \\
\leq & C\left\|\lambda^{-l}|X|^{-1 / 2+l} \sigma\left(\lambda^{-1} X, D\right) R( \pm 1 \pm i 0) \tau_{\lambda}(X, D)^{*} v\right\|_{L^{2}\left(\mathbb{R}^{n}\right)} \\
+ & C\left\|\lambda^{-l^{\prime}}|X|^{-1 / 2+l^{\prime}} \sigma\left(\lambda^{-1} X, D\right) R( \pm 1 \pm i 0) \tau_{\lambda}(X, D)^{*} v\right\|_{L^{2}\left(\mathbb{R}^{n}\right)}
\end{aligned}
$$

If $0 \leq l \leq 1 / 2$, then we have $\lambda^{-1 / 2}\left\langle\lambda^{-1} x\right\rangle^{-1 / 2+l} \leq \lambda^{-l}|x|^{-1 / 2+l}$ and

$$
\begin{aligned}
& \left\|\sigma_{\lambda}(X, D) R( \pm 1 \pm i 0) \tau_{\lambda}(X, D)^{*} v\right\|_{L^{2}\left(\mathbb{R}^{n}\right)} \\
\leq & C\left\|\lambda^{-l}|X|^{-1 / 2+l} \sigma\left(\lambda^{-1} X, D\right) R( \pm 1 \pm i 0) \tau_{\lambda}(X, D)^{*} v\right\|_{L^{2}\left(\mathbb{R}^{n}\right)} .
\end{aligned}
$$


Furthermore, since we have $\lambda^{-1 / 2}\left\langle\lambda^{-1} x\right\rangle^{-1 / 2-l} \leq \lambda^{-1 / 2}\left\langle\lambda^{-1} x\right\rangle^{-1 / 2-l^{\prime}} \leq \lambda^{l^{\prime}}|x|^{-1 / 2-l^{\prime}}$ for $l^{\prime} \leq l$, we may replace $\tau_{\lambda}(x, \xi)$ by $\lambda^{l}|x|^{-1 / 2-l} \tau\left(\lambda^{-1} x, \xi\right)$ or $\lambda^{l^{\prime}}|x|^{-1 / 2}-l^{\prime} \tau\left(\lambda^{-1} x, \xi\right)$ whichever we like, in the right hand sides of these estimates.

On account of them, it suffices to show estimates (3.7) and (3.8) for $\sigma_{\lambda}(x, \xi)$ and $\tau_{\lambda}(x, \xi)$ of the forms

$$
\sigma_{\lambda}(x, \xi)=|x|^{-1 / 2+l} \sigma\left(\lambda^{-1} x, \xi\right), \quad \tau_{\lambda}(x, \xi)=|x|^{-1 / 2-l} \tau\left(\lambda^{-1} x, \xi\right) .
$$

for $0<l<\min \{1,(n-1) / 2\}$ assuming $\sigma_{\lambda}(x, \xi)=0$ on $\Gamma$, and for $l=0$ assuming $\sigma_{\lambda}(x, \xi)=\tau_{\lambda}(x, \xi)=0$ on $\Gamma$. We remark that $\sigma_{\lambda}(x, \xi)$ and $\tau_{\lambda}(x, \xi)$ defined by (3.9) satisfy the estimates

$$
\begin{aligned}
& \left|\partial_{x}^{\alpha} \partial_{\xi}^{\beta}\left(\sigma_{\lambda}(x, \xi)\right)\right| \leq C_{\alpha \beta}|x|^{-1 / 2+l-\alpha}|\xi|^{\theta-\beta} \\
& \left|\partial_{x}^{\alpha} \partial_{\xi}^{\beta}\left(\tau_{\lambda}(x, \xi)\right)\right| \leq C_{\alpha \beta}|x|^{-1 / 2-l-\alpha}|\xi|^{1-\theta-\beta}
\end{aligned}
$$

with constants $C_{\alpha \beta}$ independent of $\lambda>0$.

We split estimate (3.7) into the following two parts:

$$
\begin{gathered}
\sup _{\lambda>0}\left\|\sigma_{\lambda}(X, D) R(1 \pm i 0)(1-\chi \circ p)(D) \tau_{\lambda}(X, D)^{*} v\right\|_{L^{2}\left(\mathbb{R}^{n}\right)} \leq C\|v\|_{L^{2}\left(\mathbb{R}^{n}\right)}, \\
\sup _{\lambda>0}\left\|\sigma_{\lambda}(X, D) R(1 \pm i 0)(\chi \circ p)(D) \tau_{\lambda}(X, D)^{*} v\right\|_{L^{2}\left(\mathbb{R}^{n}\right)} \leq C\|v\|_{L^{2}\left(\mathbb{R}^{n}\right)},
\end{gathered}
$$

where $\chi(t) \in \mathcal{C}_{0}^{\infty}\left(\mathbb{R}_{+}\right)$is a function which is equal to 1 near $t=1$. Estimates (3.8) and (3.11) are obtained if we write

$$
\begin{aligned}
& \sigma_{\lambda}(X, D) R(-1) \tau_{\lambda}(X, D)^{*}=\tilde{\sigma}_{\lambda}(X, D, Y) m(X, D, Y) \tilde{\tau}_{\lambda}(X, D, Y)^{*}, \\
& \sigma_{\lambda}(X, D) R(1 \pm i 0)(1-\chi \circ p)(D) \tau_{\lambda}(X, D)^{*}=\tilde{\sigma}_{\lambda}(X, D, Y) \tilde{m}(X, D, Y) \tilde{\tau}_{\lambda}(X, D, Y)^{*},
\end{aligned}
$$

where

$$
\tilde{\sigma}_{\lambda}(X, D, Y)=\sigma_{\lambda}(X, D)|D|^{-\theta-1 / 2}|Y|^{-l}, \quad \tilde{\tau}_{\lambda}(X, D, Y)=\tau_{\lambda}(X, D)|D|^{\theta-3 / 2}|Y|^{l},
$$

and

$$
\begin{aligned}
& m(X, D, Y)=|X|^{l}|D|^{2} R(-1)|Y|^{-l}, \\
& \tilde{m}(X, D, Y)=|X|^{l}|D|^{2} R(1 \pm i 0)(1-\chi \circ p)(D)|Y|^{-l} .
\end{aligned}
$$

All of them are $L^{2}$-bounded (uniformly in $\lambda>0$ ) by Lemma 3.3 (with $b=1 / 2$ and $\delta=1 / 2 \mp l$ ) and estimates (3.10) together with the following lemma by Kurtz and Wheeden [KW, Theorem 3]:

Lemma 3.4. Let $-n / 2<\delta<n / 2$. Then we have

$$
\begin{aligned}
& \left\||x|^{\delta} m(D) u\right\|_{L^{2}\left(\mathbb{R}^{n}\right)} \leq\left. C \sum_{|\gamma| \leq n} \sup _{\xi \in \mathbb{R}^{n}}|| \xi\right|^{|\gamma|} \partial^{\gamma} m(\xi)\left|\left\||x|^{\delta} u\right\|_{L^{2}\left(\mathbb{R}^{n}\right)},\right. \\
& \left\|\langle x\rangle^{\delta} m(D) u\right\|_{L^{2}\left(\mathbb{R}^{n}\right)} \leq\left. C \sum_{|\gamma| \leq n} \sup _{\xi \in \mathbb{R}^{n}}|| \xi\right|^{|\gamma|} \partial^{\gamma} m(\xi) \mid\left\|\langle x\rangle^{\delta} u\right\|_{L^{2}\left(\mathbb{R}^{n}\right)} .
\end{aligned}
$$

Proof. The first estimate is due to [KW]. The second estimate with $0 \leq \delta<n / 2$ is obtained from it because of the inequality $\langle x\rangle^{\delta} \leq C\left(1+|x|^{\delta}\right)$. The one with $-n / 2<\delta \leq 0$ is just the dual of it. 
We prove estimate (3.12). Let $\rho(x) \in C_{0}^{\infty}\left(\mathbb{R}^{n}\right)$ be equal to 1 near the origin and $\tilde{\chi}(t) \in C_{0}^{\infty}\left(\mathbb{R}_{+}\right)$be equal to 1 on $\operatorname{supp} \chi$. We set

$$
\begin{aligned}
& \sigma_{\lambda}^{0}(x, \xi)=\rho(x) \sigma_{\lambda}(x, \xi), \quad \sigma_{\lambda}^{1}(x, \xi)=(1-\rho(x)) \sigma_{\lambda}(x, \xi)(\tilde{\chi} \circ p)(\xi), \\
& \tau_{\lambda}^{0}(x, \xi)=\rho(x) \tau_{\lambda}(x, \xi), \quad \tau_{\lambda}^{1}(x, \xi)=(1-\rho(x)) \tau_{\lambda}(x, \xi)(\tilde{\chi} \circ p)(\xi) .
\end{aligned}
$$

By Proposition 2.2 and estimates (3.10), we have

$$
\sup _{\lambda>0}\left\|\sigma_{\lambda}^{1}(X, D) R(1 \pm i 0)(\chi \circ p)(D) \tau_{\lambda}^{1}(X, D)^{*} v\right\|_{L^{2}\left(\mathbb{R}^{n}\right)} \leq C\|v\|_{L^{2}\left(\mathbb{R}^{n}\right)} .
$$

Other estimates are obtained form the following lemma which was proved by [SuT, Theorem 1.2] (see also [Su1, Theorem 3.1]):

Lemma 3.5. Let $1-n / 2<\alpha<1 / 2$ and $1-n / 2<\beta<1 / 2$. Then we have

$$
\left\||x|^{\alpha-1}|D|^{\alpha+\beta} R(1 \pm i 0)|x|^{\beta-1} v\right\|_{L^{2}\left(\mathbb{R}^{n}\right)} \leq C\|v\|_{L^{2}\left(\mathbb{R}^{n}\right)} .
$$

In fact, from this lemma and Lemma 3.4 with $m(\xi)=f(|\xi|)|\xi|^{-(\alpha+\beta)}(\chi \circ p)(\xi)^{-1}$, we obtain the estimate

$$
\left\||x|^{\alpha-1} R(1 \pm i 0) f(|D|)|x|^{\beta-1} v\right\|_{L^{2}\left(\mathbb{R}^{n}\right)} \leq C_{f}\|v\|_{L^{2}\left(\mathbb{R}^{n}\right)}
$$

for $f \in C_{0}^{\infty}\left(\mathbb{R}_{+}\right)$. Since $\sigma_{\lambda}^{0}(X, D)|D|^{-(\theta+\varepsilon)}|Y|^{1 / 2+\varepsilon}$ and $\tau_{\lambda}^{0}(X, D)|D|^{-(1-\theta+\varepsilon)}|Y|^{1 / 2+\varepsilon}$ are $L^{2}$-bounded uniformly in $\lambda>0$ by Lemma 3.3 (with $b=\varepsilon$ and $\delta=1 / 2+2 \epsilon$ ), where $0<\varepsilon<(n-1) / 4$ and $l / 2 \leq \varepsilon$, the estimate

$$
\sup _{\lambda>0}\left\|\sigma_{\lambda}^{0}(X, D) R(1 \pm i 0)(\chi \circ p)(D) \tau_{\lambda}^{0}(X, D)^{*} v\right\|_{L^{2}\left(\mathbb{R}^{n}\right)} \leq C\|v\|_{L^{2}\left(\mathbb{R}^{n}\right)}
$$

is reduced to the estimate

$$
\left\||x|^{-(1 / 2+\varepsilon)} R(1 \pm i 0)(\chi \circ p)(D)|D|^{1+2 \varepsilon}|x|^{-(1 / 2+\varepsilon)} v\right\|_{L^{2}\left(\mathbb{R}^{n}\right)} \leq C\|v\|_{L^{2}\left(\mathbb{R}^{n}\right)}
$$

which is a special case of estimate (3.14) with $\alpha=\beta=1 / 2-\varepsilon$. Similarly, we have that $\tau_{\lambda}^{1}(X, D)|D|^{-(1-\theta+\varepsilon)}|Y|^{1 / 2+\varepsilon}$ with $\epsilon=l / 2$ is $L^{2}$-bounded uniformly in $\lambda>0$, and the estimate

$$
\sup _{\lambda>0}\left\|\sigma_{\lambda}^{0}(X, D) R(1 \pm i 0)(\chi \circ p)(D) \tau_{\lambda}^{1}(X, D)^{*} v\right\|_{L^{2}\left(\mathbb{R}^{n}\right)} \leq C\|v\|_{L^{2}\left(\mathbb{R}^{n}\right)}
$$

with $l>0$ is also reduced to estimate (3.14).

Hence all the rest to be shown is the estimate

$$
\sup _{\lambda>0}\left\|\sigma_{\lambda}^{1}(X, D) R(1 \pm i 0)(\chi \circ p)(D) \tau_{\lambda}^{0}(X, D)^{*} v\right\|_{L^{2}\left(\mathbb{R}^{n}\right)} \leq C\|v\|_{L^{2}\left(\mathbb{R}^{n}\right)} .
$$

We remark that the estimate (3.16) with $l=0$ is just the dual of estimate (3.17) with $l=0$. By Lemmas 2.3 and Lemma 2.1, estimate (3.17) is reduced to the estimate

$$
\sup _{\lambda>0}\left\|\langle x\rangle^{-3 / 2+l} R(1 \pm i 0)(\chi \circ p)(D)\left(\Omega_{i j}\right)^{k} \tau_{\lambda}^{0}(X, D)^{*} v\right\|_{L^{2}\left(\mathbb{R}^{n}\right)} \leq C\|v\|_{L^{2}\left(\mathbb{R}^{n}\right)},
$$

where $k=0,1$. Since the symbol of $\Omega_{i j}$ is linear in $x$ and positively homogeneous of order 1 in $\xi$ by (2.2),$\tau_{\lambda}^{0}(X, D)\left(\Omega_{i j}^{*}\right)^{k}$ is a finite sum of the operators of the form $\rho(X)|X|^{-1 / 2-l+\mu} \tilde{\tau}_{\lambda}(X, D)|D|^{\mu}$, where $\mu=0,1$ and $\tilde{\tau}_{\lambda}(x, \xi)$ is homogeneous of orders 
$1-\theta$ in $\xi$. Furthermore $\rho(X)|X|^{-1 / 2-l+\mu} \tilde{\tau}_{\lambda}(X, D)|D|^{\mu}|D|^{-(1-\theta+\varepsilon+\mu)}|Y|^{1 / 2+\varepsilon}$ are $L^{2}$ bounded uniformly in $\lambda>0$ by Lemma 3.3 (with $b=\varepsilon$ and $\delta=1 / 2+2 \epsilon$ ), where $0<\varepsilon<(n-1) / 4$ and $l / 2 \leq \varepsilon$. Noticing the trivial inequality $\langle x\rangle^{-3 / 2+l} \leq\langle x\rangle^{l / 2-1} \leq$ $|x|^{l / 2-1}$, the estimate is further reduced to

$$
\left\||x|^{l / 2-1} R(1 \pm i 0)(\chi \circ p)(D)|D|^{1-\theta+\varepsilon+\mu}|x|^{-(1 / 2+\varepsilon)} v\right\|_{L^{2}\left(\mathbb{R}^{n}\right)} \leq C\|v\|_{L^{2}\left(\mathbb{R}^{n}\right)}
$$

which are implied again by (3.14) with $\alpha=l / 2$ and $\beta=1 / 2-\varepsilon$.

Summing up estimates (3.13), (3.15), (3.16), and (3.17), we have estimate (3.12), and thus the proof of Theorem 3.1 is complete.

\section{Herbst-Skibsted's Resolvent estimate}

In this section, we will explain the relation between Theorem 3.1 and HerbstSkibsted's resolvent estimate in [HS].

Let $S(x, \lambda)$ be the solution of eikonal equation,

$$
p(\nabla S(x, \lambda))^{2}+V(x)=\lambda \quad(\lambda>0)
$$

for $L=p(D)^{2}+V(x)$. Here we always assume $V=0$ but keep it remaining in the notation because the case $V \neq 0$ is admitted in [HS] assuming the potential $V$ to be smooth and to have the property

$$
\left|\partial^{\alpha} V(x)\right| \leq C_{\alpha}\langle x\rangle^{-\varepsilon_{0}-|\alpha|}
$$

where $0<\varepsilon_{0}<1$. In this case, we have $S(x, \lambda)=\sqrt{\lambda} p^{*}(x)$, where $p^{*}(x)$ is the dual function of $p(\xi)$ defined by satisfying the relations $p\left(\nabla p^{*}(x)\right)=1$ and $\nabla p^{*}(\nabla p(\xi))=$ $\xi / p(\xi)$ (see [RS2, Theorem 3.1]). Noting that $D=-i \nabla$ is the momentum operator, we set

$$
\gamma(\lambda)=D \mp \nabla S(x, \lambda) .
$$

The quantisation of $\gamma(\lambda)$ is given by

$$
\bar{\gamma}=D \mp \nabla S\left(x, p(D)^{2}\right) .
$$

We remark that the symbol $\bar{\gamma}(x, \xi)=\xi \mp p(\xi) \nabla p^{*}(x)$ of the operator $\bar{\gamma}$ satisfies the half structure condition

$$
\bar{\gamma}(x, \xi)=0 \text { on } \Gamma^{ \pm},
$$

where

$$
\Gamma^{ \pm}=\left\{(\lambda \nabla p(\xi), \xi): x \in \mathbb{R}^{n} \backslash 0, \pm \lambda>0\right\} .
$$

In the case $L=-\Delta$, for example, we have

$$
\gamma(\lambda)=D \mp \sqrt{\lambda} \frac{x}{|x|}, \quad \bar{\gamma}=D \mp \frac{x}{|x|}|D|,
$$

and the following results are already known, as an adapted version of those in [HS]:

Theorem 4.1 ([S, Theorems 4.4, 5.1]). Let $0 \leq s \leq 1$, let $\delta>1 / 2$, and let $\chi \in C_{0}^{\infty}\left(\mathbb{R}_{+}\right)$. Assume $L=-\Delta+V$. Then, we have a quantum result

$$
\sup _{\lambda \in \mathbb{R}}\|\bar{\gamma} R(\lambda \pm i 0) \chi(|D|) v\|_{L_{-\delta+s}^{2}} \leq C\|v\|_{L_{\delta+s}^{2}}
$$

and a classical result $\gamma(\lambda) R(\lambda \pm i 0) \in \mathcal{L}\left(L_{\delta+s}^{2}, L_{-\delta+s}^{2}\right)$. 
The quantum result in Theorem 4.1 with $s=0$ is a usual resolvent estimate, but we can extend it to the case $0<s \leq 1$ by virtue of the half structure (4.1) of the operator $\bar{\gamma}$. We remark that the classical result in Theorem 4.1 was first proved by Isozaki [I], and it can be also derived from the quantum result in Theorem 4.1. Furthermore, it implies Sommerfeld's radiation condition

$$
\left(\partial_{r} \mp i \sqrt{\lambda}\right) u \in L_{-\delta+s}^{2}
$$

for the outgoing and incoming solutions $u=R(\lambda \pm i 0) v$ to the Helmholtz equation

$$
(-\triangle-\lambda) u=v, \quad\left(\lambda>0, v \in L_{\delta+s}^{2}\right)
$$

since $i(x /|x|) \cdot \gamma(\lambda)=\partial_{r} \mp i \sqrt{\lambda}$.

Theorem 4.1 means that each specified operator $\sigma^{ \pm}(X, D)=\bar{\gamma}$ with half structure $\sigma^{ \pm}(x, \xi)=0$ on $\Gamma^{ \pm}$implies the estimates for $R(\lambda+i 0)$ and $R(\lambda-i 0)$, respectively. On the other hand, our Theorem 3.1 means that any operator $\sigma(X, D)$ with full structure $\sigma(x, \xi)=0$ on $\Gamma$ implies estimates for both.

Furthermore, Theorem 3.1 corresponds to the critical case of Theorem 4.1. In fact, the quantum estimate in Theorem 4.1 can be interpreted as

$$
\sup _{\lambda \in \mathbb{R}}\left\|\sigma^{ \pm}(X, D) R(\lambda \pm i 0) \chi(|D|) v\right\|_{L_{-1 / 2+l}^{2}} \leq C\|v\|_{L_{1 / 2+l+2 \varepsilon}^{2}} \quad(-\varepsilon \leq l<1, \varepsilon>0)
$$

for $\sigma^{ \pm}(X, D)=\bar{\gamma}$, while estimate (3.4) in Corollary 3.2 (together with Lemma 3.4) implies a better estimate

$$
\sup _{\lambda \in \mathbb{R}}\|\sigma(X, D) R(\lambda \pm i 0) \chi(|D|) v\|_{L_{-1 / 2+l}^{2}} \leq C\|v\|_{L_{1 / 2+l}^{2}} \quad(0<l<1)
$$

for $\sigma(X, D)$ satisfying the full structure condition (in the case $n \geq 3$ ).

As another advantage of Theorem 3.1, we can treat the general operator $L=$ $p(D)^{2}+V$ instead of $-\Delta+V$ although we have to assume $V=0$.

\section{Smoothing EStimates For Schrödinger EQUATions}

It is known that uniform resolvent estimates straightforwardly induce smoothing estimates for Schorödinger evolution operators. For example, estimate (3.3) in Corollary 3.2 says that the operator $A=\langle x\rangle^{-1 / 2} \sigma(X, D)$ is $L$-supersmooth on the separable Hilbert space $H=L^{2}$, that is, $A$ satisfies

$$
\sup _{\lambda \in \mathbb{R}}\left\|A R(\lambda \pm i 0) A^{*} v\right\|_{H} \leq C\|v\|_{H} .
$$

Then by the work of Kato [K], we have the estimate

$$
\int\|A u\|_{H}^{2} d t \leq C\|\varphi\|_{H}^{2}
$$

for the solution $u(t, x)=e^{-i t L} \varphi(x)$ to

$$
\left\{\begin{aligned}
\left(i \partial_{t}-L\right) u(t, x) & =0 \\
u(0, x) & =\varphi(x) .
\end{aligned}\right.
$$

Hence we equivalently have 
Theorem 5.1. Let $n \geq 2$. Suppose that $\sigma(x, \xi) \sim|\xi|^{1 / 2}$ and $\sigma(x, \xi)=0$ on $\Gamma$. Then the solution $u$ to equation (5.1) satisfies the estimate

$$
\left\|\langle x\rangle^{-1 / 2} \sigma(X, D) u\right\|_{L^{2}\left(\mathbb{R}_{t} \times \mathbb{R}_{x}^{n}\right)} \leq C\|\varphi\|_{L^{2}\left(\mathbb{R}_{x}^{n}\right)} .
$$

We remark that Theorem 5.1 is a refinement of the following well known smoothing estimate

$$
\left\|\langle x\rangle^{-1 / 2-l}|D|^{1 / 2} u\right\|_{L^{2}\left(\mathbb{R}_{t} \times \mathbb{R}_{x}^{n}\right)} \leq C\|\varphi\|_{L^{2}\left(\mathbb{R}_{x}^{n}\right)} \quad(l>0)
$$

for the solution $u$ to equation (5.1) (see, for example, BK] and [Ch2]). Theorem 5.1 covers its critical case $l=0$ under the structure condition $\sigma(x, \xi)=0$ on $\Gamma$. In our previous work [RS2], we also proved the estimate

$$
\left\||x|^{-1 / 2} \sigma(X, D) u\right\|_{L^{2}\left(\mathbb{R}_{t} \times \mathbb{R}_{x}^{n}\right)} \leq C\|\varphi\|_{L^{2}\left(\mathbb{R}_{x}^{n}\right)}
$$

when $\sigma(x, \xi)$ satisfies the same structure condition, and is positively homogeneous of order 0 in $x$ and $1 / 2$ in $\xi$. Estimate (5.3) is a refinement of the estimate

$$
\left\||x|^{\alpha-1}|D|^{\alpha} u\right\|_{L^{2}\left(\mathbb{R}_{t} \times \mathbb{R}_{x}^{n}\right)} \leq C\|\varphi\|_{L^{2}\left(\mathbb{R}_{x}^{n}\right)} \quad(1-n / 2<\alpha<1 / 2)
$$

by $[\mathrm{KY}]$ and $[\mathrm{Su} 1$.

On the other hand, as it is discussed in [Su1], Su2], [SuT], we can construct the solution $u(t, x)$ to the inhomogeneous equation

$$
\left\{\begin{aligned}
\left(i \partial_{t}-L\right) u(t, x) & =f(t, x) \\
u(0, x) & =0
\end{aligned}\right.
$$

as

$$
u(t, x)=\frac{1}{i} \mathcal{F}_{\tau}^{-1} R(-\tau+i 0) \mathcal{F}_{t} f^{+}(t, x)+\frac{1}{i} \mathcal{F}_{\tau}^{-1} R(-\tau-i 0) \mathcal{F}_{t} f^{-}(t, x) .
$$

Here $f^{ \pm}$denotes the function $f$ multiplied by the Heaviside function $Y( \pm t)$, that is, the characteristic function of the set $\{t: \pm t \geq 0\}$. Hence estimate (3.4) in Corollary 3.2 yields the following result for (5.4), which is a refinement of the estimate

$$
\left\|\langle x\rangle^{-1 / 2-l}|D| u\right\|_{L^{2}\left(\mathbb{R}_{t} \times \mathbb{R}_{x}^{n}\right)} \leq C\left\|\langle x\rangle^{1 / 2+l} f\right\|_{L^{2}\left(\mathbb{R}_{t} \times \mathbb{R}_{x}^{n}\right)} \quad(l>0)
$$

(see, for example, Ch2]):

Theorem 5.2. Let $n \geq 2$. Suppose that $\sigma(x, \xi) \sim|\xi|$ and $\sigma(x, \xi)=0$ on $\Gamma$. Then the solution $u$ to equation (5.4) satisfies the estimate

$$
\left\|\langle x\rangle^{-1 / 2+l} \sigma(X, D) u\right\|_{L^{2}\left(\mathbb{R}_{t} \times \mathbb{R}_{x}^{n}\right)} \leq C\left\|\langle x\rangle^{1 / 2+l} f\right\|_{L^{2}\left(\mathbb{R}_{t} \times \mathbb{R}_{x}^{n}\right)}
$$

for $0<l<1$ if $n \geq 3$ and $0<l<1 / 2$ if $n=2$.

If we drop the structure assumption $\sigma(x, \xi)=0$ on $\Gamma$ from Theorems [5.1 5.2 , we cannot expect the same estimates there but can still show the following weaker ones: 
Proposition 5.3. Let $n \geq 2$ and let $l>0$. Suppose that $\sigma(x, \xi) \sim|\xi|^{1 / 2}$. Then the solution $u$ to equation (5.1) satisfies the estimate

$$
\left\|\langle x\rangle^{-1 / 2-l} \sigma(X, D) u\right\|_{L^{2}\left(\mathbb{R}_{t} \times \mathbb{R}_{x}^{n}\right)} \leq C\|\varphi\|_{L^{2}\left(\mathbb{R}_{x}^{n}\right)} .
$$

Suppose that $\sigma(x, \xi) \sim|\xi|$. Then the solution $u$ to equation (5.4) satisfies the estimate

$$
\left\|\langle x\rangle^{-1 / 2-l} \sigma(X, D) u\right\|_{L^{2}\left(\mathbb{R}_{t} \times \mathbb{R}_{x}^{n}\right)} \leq C\left\|\langle x\rangle^{1 / 2+l} f\right\|_{L^{2}\left(\mathbb{R}_{t} \times \mathbb{R}_{x}^{n}\right)} .
$$

Proof. We may assume $0<l<(n-1) / 2$ since the estimate with $l \geq(n-1) / 2$ is a weaker result. Let $0<l^{\prime}<l<(n-1) / 2$ and let us factorise $\langle x\rangle^{-1 / 2-l} \sigma(X, D)$ as

$$
\begin{aligned}
\langle x\rangle^{-1 / 2-l} \sigma(X, D) & =\langle x\rangle^{-1 / 2-l} \sigma(X, D)|D|^{-1 / 2}\langle x\rangle^{1 / 2+l^{\prime}} \cdot\langle x\rangle^{-1 / 2-l^{\prime}}|D|^{1 / 2} \\
& =\langle x\rangle^{-1 / 2-l} \sigma(X, D)|D|^{-1}\langle x\rangle^{1 / 2+l^{\prime}} \cdot\langle x\rangle^{-1 / 2-l^{\prime}}|D| .
\end{aligned}
$$

On account of estimates (5.2) and (5.5), it suffices to show the $L^{2}$-boundedness of the operator $\langle x\rangle^{-1 / 2-l} \sigma(X, D)\langle x\rangle^{1 / 2+l^{\prime}}$ assuming $\sigma(x, \xi) \sim|\xi|^{0}$. Let $\chi(\xi) \in \mathcal{C}_{0}^{\infty}\left(\mathbb{R}^{n}\right)$ be a function which is equal to 1 near the origin. Then by the symbolic calculus and the $L^{2}$-boundedness of pseudo-differential operators of class $S^{0}$ (see also [RS1, Theorem 1.1]), the operator $\langle x\rangle^{-1 / 2-l} \sigma(X, D)(1-\chi)(D)\langle x\rangle^{1 / 2+l^{\prime}}$ is $L^{2}$-bounded. On the other hand, the $L^{2}$-boundedness of the operator $\langle x\rangle^{-1 / 2-l} \sigma(X, D) \chi(D)\langle x\rangle^{1 / 2+l^{\prime}}$ is reduced to that of $|x|^{-1 / 2-l} \sigma(X, D) \chi(D)|x|^{1 / 2+l^{\prime}}$ and $|x|^{-1 / 2-l} \sigma(X, D) \chi(D)$ since $\langle x\rangle^{-(1 / 2+l)} \leq$ $|x|^{-(1 / 2+l)}$ and $\langle x\rangle^{1 / 2+l^{\prime}} \leq C\left(1+|x|^{1 / 2+l^{\prime}}\right)$. Due to Lemma 3.4, they are further reduced to that of $|x|^{-1 / 2-l} \sigma(X, D)|D|^{-\left(l-l^{\prime}\right)}|x|^{1 / 2+l^{\prime}}$ and $|x|^{-1 / 2-l} \sigma(X, D)|D|^{-1 / 2-l}$, which are obtained from Lemma 3.3 with $b=l-l^{\prime}, \delta=1 / 2+l$ and $b=1 / 2+l, \delta=1 / 2+l$, respectively.

We have also a result similar to Theorem 5.2 for the solution to homogeneous equation (5.1):

Theorem 5.4. Let $n \geq 3$. Suppose that $\sigma(x, \xi) \sim|\xi|$ and $\sigma(x, \xi)=0$ on $\Gamma$. Then the solution $u$ to equation (5.1) satisfies the estimate

$$
\left\|\langle x\rangle^{-1 / 2+l} \sigma(X, D) u\right\|_{L^{2}\left(\mathbb{R}_{t} \times \mathbb{R}_{x}^{n}\right)} \leq C\left\|\langle x\rangle^{\alpha l}\langle D\rangle^{1 / 2} \varphi\right\|_{L^{2}\left(\mathbb{R}_{x}^{n}\right)}
$$

for $0<l<1$ and $\alpha>3 / 2$.

Proof. We decompose the solution $u=e^{-i t L} \varphi$ into the following two parts:

$$
u_{\text {low }}=e^{-i t L} \chi(L) \varphi, \quad u_{\text {high }}=e^{-i t L}(1-\chi(L)) \varphi,
$$

where $\chi \in C_{0}^{\infty}(\mathbb{R})$ is a function such that $\chi(\xi)=1$ for $|\xi|<1$, and $\chi(L)=\left(\chi \circ p^{2}\right)(D)$. Let $\tilde{\chi} \in C_{0}^{\infty}(\mathbb{R})$ be another function such that $\tilde{\chi}(\xi)=1$ on $\operatorname{supp} \chi$. Then we have $u_{\text {low }}=e^{-i t L} \chi(L) \tilde{\varphi}$, where $\tilde{\varphi}=\left(\tilde{\chi} \circ p^{2}\right)(D) \varphi$. Let

$$
L=\int \lambda d E_{L}(\lambda)=\int \lambda A_{L}(\lambda) d \lambda
$$


be the spectral decomposition of the self-adjoint operator $L$ with spectral measure $E_{L}(\lambda)$, and

$$
A_{L}(\lambda)=\frac{d E_{L}(\lambda)}{d \lambda}=\frac{1}{2 \pi i}(R(\lambda+i 0)-R(\lambda-i 0))
$$

be the corresponding spectral density. Then we can write

$$
u_{\text {low }}=e^{-i t L} \chi(L) \tilde{\varphi}=\int e^{-i t \lambda} \chi(\lambda) A_{L}(\lambda) \tilde{\varphi} d \lambda,
$$

and by Plancherel's theorem (see also [ReS, Section XIII.7, Lemma 1]), estimate (3.4) in Corollary 3.2, and Lemma 3.4, we have

$$
\begin{aligned}
& \left\|\langle x\rangle^{-1 / 2+l} \sigma(X, D) u_{\text {low }}\right\|_{L^{2}\left(\mathbb{R}_{t} \times \mathbb{R}_{x}^{n}\right)}^{2} \\
= & 2 \pi \int|\chi(\lambda)|^{2}\left\|\langle x\rangle^{-1 / 2+l} \sigma(X, D) A_{L}(\lambda) \tilde{\varphi}\right\|_{L^{2}\left(\mathbb{R}_{x}^{n}\right)}^{2} d \lambda \\
\leq & C \sup _{\lambda \in \mathbb{R}}\|\sigma(X, D)(R(\lambda+i 0)-R(\lambda-i 0)) \tilde{\varphi}\|_{L_{-1 / 2+l}^{2}}^{2}\left(\mathbb{R}_{x}^{n}\right) \\
\leq & C\|\tilde{\varphi}\|_{L_{1 / 2+l}^{2}}^{2}\left(\mathbb{R}_{x}^{n}\right) \leq C\left\|\langle x\rangle^{1 / 2+l}\langle D\rangle^{1 / 2} \varphi\right\|_{L^{2}\left(\mathbb{R}_{x}^{n}\right)}^{2} .
\end{aligned}
$$

On the other hand, by Lemma 2.3, Proposition 5.3, Lemma 2.5, and Lemma 3.4, we have

$$
\begin{aligned}
& \left\|\langle x\rangle^{-1 / 2+l} \sigma(X, D) u_{\text {high }}\right\|_{L^{2}\left(\mathbb{R}_{t} \times \mathbb{R}_{x}^{n}\right)}=\left\|\langle x\rangle^{-1 / 2+l} \sigma(X, D)(1-\chi(L)) e^{-i t L} \varphi\right\|_{L^{2}\left(\mathbb{R}_{t} \times \mathbb{R}_{x}^{n}\right)} \\
\leq & C\left(\sum_{i<j}\left\|\langle x\rangle^{-1 / 2-(1-l)} e^{-i t L} \Omega_{i j}(X, D) \varphi\right\|_{L^{2}\left(\mathbb{R}_{t} \times \mathbb{R}_{x}^{n}\right)}+\left\|\langle x\rangle^{-1 / 2-(1-l)} e^{-i t L} \varphi\right\|_{L^{2}\left(\mathbb{R}_{t} \times \mathbb{R}_{x}^{n}\right)}\right) \\
\leq & C\left(\sum_{i<j}\left\|\Omega_{i j}(X, D) p(D)^{-1 / 2} \varphi\right\|_{L^{2}\left(\mathbb{R}_{x}^{n}\right)}+\left\||D|^{-1 / 2} \varphi\right\|_{L^{2}\left(\mathbb{R}_{x}^{n}\right)}\right) \\
\leq & C\left\|\langle x\rangle\langle D\rangle^{1 / 2} \varphi\right\|_{L^{2}\left(\mathbb{R}_{x}^{n}\right)}
\end{aligned}
$$

since $\Omega_{i j}(X, D)$ commutes with $e^{-i t L}$ and $p(D)^{-1 / 2}$ by Lemma 2.1 . Hence, for $1 / 2 \leq$ $l<1$, we have

$$
\left\|\langle x\rangle^{-1 / 2+l} \sigma(X, D) u\right\|_{L^{2}\left(\mathbb{R}_{t} \times \mathbb{R}_{x}^{n}\right)} \leq C\left\|\langle x\rangle^{1 / 2+l}\langle D\rangle^{1 / 2} \varphi\right\|_{L^{2}\left(\mathbb{R}_{x}^{n}\right)} .
$$

The conclusion of the theorem is obtained if we interpolate this estimate and the estimate

$$
\left\|\langle x\rangle^{-1 / 2} \sigma(X, D) u\right\|_{L^{2}\left(\mathbb{R}_{t} \times \mathbb{R}_{x}^{n}\right)} \leq C\left\|\langle D\rangle^{1 / 2} \varphi\right\|_{L^{2}\left(\mathbb{R}_{x}^{n}\right)}
$$

which is a direct consequence of Theorem 5.1 and Lemma 3.4 .

For $s, \tilde{s} \in \mathbb{R}$, let $H^{s, \tilde{s}}\left(\mathbb{R}_{t} \times \mathbb{R}_{x}^{n}\right)$ be the set of tempered distributions $g$ on $\mathbb{R}_{t} \times \mathbb{R}_{x}^{n}$ such that the norm

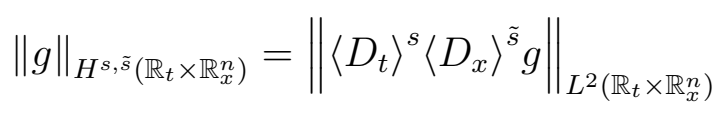


is finite, where $\left\langle D_{t}\right\rangle^{s}=\mathcal{F}_{\tau}^{-1}\langle\tau\rangle^{s} \mathcal{F}_{t}$ and $\left\langle D_{x}\right\rangle^{\tilde{s}}=\mathcal{F}_{\xi}^{-1}\langle\xi\rangle^{\tilde{s}} \mathcal{F}_{x}$. Combining Theorems 5.2 and 5.4, we have the following result:

Corollary 5.5. Let $n \geq 3$, let $0 \leq s \leq 1$, and let $\tilde{s} \geq 0$. Suppose that $\sigma(x, \xi) \sim|\xi|$ and $\sigma(x, \xi)=0$ on $\Gamma$. Then the solution $u$ to equation

$$
\left\{\begin{aligned}
\left(i \partial_{t}-L\right) u(t, x) & =f(t, x), \\
u(0, x) & =\varphi(x),
\end{aligned}\right.
$$

satisfies the estimate

$$
\begin{aligned}
& \left\|\langle x\rangle^{-1 / 2+l} \sigma(X, D) u\right\|_{H^{s, \tilde{s}}\left(\mathbb{R}_{t} \times \mathbb{R}_{x}^{n}\right)} \\
& \leq C\left(\left\|\langle x\rangle^{\alpha l}\langle D\rangle^{2 s+\tilde{s}+1 / 2} \varphi\right\|_{L^{2}\left(\mathbb{R}_{x}^{n}\right)}+\left\|\langle x\rangle^{1 / 2+l} f\right\|_{H^{s, \tilde{s}\left(\mathbb{R}_{t} \times \mathbb{R}_{x}^{n}\right)}}\right)
\end{aligned}
$$

for $0<l<1$ and $\alpha>3 / 2$.

Proof. Differentiating equation (5.6), we have

$$
\left\{\begin{aligned}
\left(i \partial_{t}-L\right) D_{x}^{k} u(t, x) & =D_{x}^{k} f(t, x), \\
D_{x}^{k} u(0, x) & =D^{k} \varphi(x),
\end{aligned}\right.
$$

and

$$
\left\{\begin{aligned}
\left(i \partial_{t}-L\right) D_{t} D_{x}^{k} u(t, x) & =D_{t} D_{x}^{k} f(t, x), \\
D_{t} D_{x}^{k} u(0, x) & =-L D^{k} \varphi(x)-D_{x}^{k} f(0, x),
\end{aligned}\right.
$$

for non-negative integers $k$. Then by Theorems 5.2 and 5.4, we have the estimate

$$
\begin{gathered}
\left\|\langle x\rangle^{-1 / 2+l} \sigma(X, D) D_{t}^{j} D_{x}^{k} u\right\|_{L^{2}\left(\mathbb{R}_{x} \times \mathbb{R}_{x}^{n}\right)} \\
\leq C\left(\left\|\langle x\rangle^{\alpha l}\langle D\rangle^{2 j+k+1 / 2} \varphi\right\|_{L^{2}\left(\mathbb{R}_{x}^{n}\right)}+\left\|\langle x\rangle^{1 / 2+l}\left\langle D_{t}\right\rangle^{j}\left\langle D_{x}\right\rangle^{k} f(t, x)\right\|_{L^{2}\left(\mathbb{R}_{t} \times \mathbb{R}_{x}^{n}\right)}\right) \\
\leq C\left(\left\|\langle x\rangle^{\alpha l}\langle D\rangle^{2 j+k+1 / 2} \varphi\right\|_{L^{2}\left(\mathbb{R}_{x}^{n}\right)}+\left\|\langle x\rangle^{1 / 2+l} f(t, x)\right\|_{H^{j, k}\left(\mathbb{R}_{t} \times \mathbb{R}_{x}^{n}\right)}\right),
\end{gathered}
$$

for $j=0,1$. Here we have used Lemma 3.4. Sobolev's embedding $H^{1}\left(\mathbb{R}_{t}\right) \hookrightarrow L^{\infty}\left(\mathbb{R}_{t}\right)$, and the $L^{2}$-boundedness of the operator $\langle x\rangle^{1 / 2+l}\langle D\rangle^{k}\langle x\rangle^{-(1 / 2+l)}\langle D\rangle^{-k}$ which can be justified by the symbolic calculus and the $L^{2}$-boundedness of pseudo-differential operators of class $S^{0}$. On the other hand, the commutator $\left[D_{x}^{k},\langle x\rangle^{-1 / 2+l} \sigma(X, D)\right]$ is again a pseudo-differential operator with a symbol of the form $\langle x\rangle^{-1 / 2-(1-l)} \tau(x, \xi)$ where $\tau(x, \xi) \sim|\xi|^{k^{\prime}}\left(0 \leq k^{\prime} \leq k\right)$. Hence, if we use Proposition 5.3 instead, we have similarly the estimate

$$
\begin{aligned}
& \left\|\left[D_{x}^{k},\langle x\rangle^{-1 / 2+l} \sigma(X, D)\right] D_{t}^{j} u\right\|_{L^{2}\left(\mathbb{R}_{t} \times \mathbb{R}_{x}^{n}\right)} \\
\leq & C\left(\left\|\langle D\rangle^{2 j+k-1 / 2} \varphi\right\|_{L^{2}\left(\mathbb{R}_{x}^{n}\right)}+\left\|\langle x\rangle^{1 / 2+l} f(t, x)\right\|_{H^{j, k}\left(\mathbb{R}_{t} \times \mathbb{R}_{x}^{n}\right)}\right) \\
\leq & C\left(\left\|\langle x\rangle^{l}\langle D\rangle^{2 j+k+1 / 2} \varphi\right\|_{L^{2}\left(\mathbb{R}_{x}^{n}\right)}+\left\|\langle x\rangle^{1 / 2+l} f(t, x)\right\|_{H^{j, k}\left(\mathbb{R}_{t} \times \mathbb{R}_{x}^{n}\right)}\right)
\end{aligned}
$$


since $1-l, l>0$. Thus we have the desired estimate if $s, \tilde{s}$ are integers. By interpolation, we have the conclusion.

\section{Derivative Nonlinear Schrödinger Equations With Structure}

Estimates obtained in the previous section can be used to show a time global existence result for derivative nonlinear Schrödinger equations. We consider the power of the derivative $\sigma(X, D) u$ of $u(t, x)$ in the space variable $x$ as nonlinear term.

Theorem 6.1. Let $n \geq 3$, let $N \geq 4, N \in \mathbb{N}$, let $\kappa>3(N+1) / 4(N-1)$, and let $\tau>(n+3) / 2$. Suppose that $\sigma(x, \xi) \sim|\xi|$ and $\sigma(x, \xi)=0$ on $\Gamma$. Assume that $\langle x\rangle^{\kappa}\langle D\rangle^{\tau} \varphi \in L^{2}$ and that its $L^{2}$-norm is sufficiently small. Then the equation

$$
\left\{\begin{array}{c}
\left(i \partial_{t}-L\right) u(t, x)=(\sigma(X, D) u)^{N} \\
u(0, x)=\varphi(x), \quad t \in \mathbb{R}, x \in \mathbb{R}^{n}
\end{array}\right.
$$

has a time global solution $u \in C^{0}\left(\mathbb{R}_{t} \times \mathbb{R}_{x}^{n}\right)$.

The key point of the proof is that the space $H^{s, \tilde{s}}=H^{s, \tilde{s}}\left(\mathbb{R}_{t} \times \mathbb{R}_{x}^{n}\right)$ is an algebra if $s>1 / 2$ and $\tilde{s}>n / 2$. Then we have

$$
\begin{aligned}
\left\|\langle x\rangle^{1 / 2+l} F^{N}\right\|_{H^{s, \tilde{s}}\left(\mathbb{R}_{t} \times \mathbb{R}_{x}^{n}\right)} & \leq C\left\|\langle x\rangle^{1 /(2 N)+l / N} F\right\|_{H^{s, \tilde{s}}\left(\mathbb{R}_{t} \times \mathbb{R}_{x}^{n}\right)}^{N} \\
& \leq C\left\|\langle x\rangle^{-1 / 2+l} F\right\|_{H^{s, \tilde{s}}\left(\mathbb{R}_{t} \times \mathbb{R}_{x}^{n}\right)}^{N}
\end{aligned}
$$

if $l=(N+1) /(2 N-2)$. Note that $1 / 2<l<1$ if $N \geq 4$. Using the formula $F^{N}-G^{N}=(F-G)\left(F^{N-1}+F^{N-2} G+\cdots+G^{N-1}\right)$, we have similarly

$$
\begin{aligned}
\left\|\langle x\rangle^{1 / 2+l}\left(F^{N}-G^{N}\right)\right\|_{H^{s, \tilde{s}}\left(\mathbb{R}_{t} \times \mathbb{R}_{x}^{n}\right)} & \\
\leq C\left(\sum_{j=0}^{N-1}\left\|\langle x\rangle^{-1 / 2+l} F\right\|_{H^{s, \tilde{s}}}^{N-1-j} \cdot\right. & \left.\left\|\langle x\rangle^{-1 / 2+l} G\right\|_{H^{s, \tilde{s}}}^{j}\right) \\
& \times\left\|\langle x\rangle^{-1 / 2+l}(F-G)\right\|_{H^{s, \tilde{s}}\left(\mathbb{R}_{t} \times \mathbb{R}_{x}^{n}\right)} .
\end{aligned}
$$
for

$$
\left\{\begin{array}{c}
\left(i \partial_{t}-L\right) u(t, x)=\left(\sigma(X, D) u_{0}\right)^{N} \\
u(0, x)=\varphi(x), \quad t \in \mathbb{R}, x \in \mathbb{R}^{n} .
\end{array}\right.
$$

We take $1 / 2<s \leq 1$ and $\tilde{s}>n / 2$ such that $\tau=2 s+\tilde{s}+1 / 2$, and let us use Corollary 5.5 with $f=\left(\sigma(X, D) u_{0}\right)^{N}$ and $l=(N+1) /(2 N-2)$. On account of (6.2), we have an estimate

$$
\begin{aligned}
& \left\|\langle x\rangle^{-1 / 2+l} \sigma(X, D) u\right\|_{H^{s, \tilde{s}}\left(\mathbb{R}_{t} \times \mathbb{R}_{x}^{n}\right)} \\
& \quad \leq C\left(\left\|\langle x\rangle^{\kappa}\langle D\rangle^{\tau} \varphi\right\|_{L^{2}\left(\mathbb{R}_{x}^{n}\right)}+\left\|\langle x\rangle^{-1 / 2+l} \sigma(X, D) u_{0}\right\|_{H^{s, \tilde{s}}\left(\mathbb{R}_{t} \times \mathbb{R}_{x}^{n}\right)}^{N}\right)
\end{aligned}
$$


for $\kappa>(3 / 2) l$. On the other hand, let $\tilde{u}$ be the solution of the equation

$$
\left\{\begin{array}{c}
\left(i \partial_{t}-L\right) \tilde{u}(t, x)=\left(\sigma(X, D) \tilde{u}_{0}\right)^{N} \\
u(0, x)=\varphi(x), \quad t \in \mathbb{R}, x \in \mathbb{R}^{n}
\end{array}\right.
$$

Then by Corollary 5.5 with $\varphi=0$, we have

$$
\left\|\langle x\rangle^{-1 / 2+l} \sigma(X, D)(u-\tilde{u})\right\|_{H^{s, \tilde{s}}\left(\mathbb{R}_{t} \times \mathbb{R}_{x}^{n}\right)} \leq C\left\|\langle x\rangle^{1 / 2+l}(f-\tilde{f})\right\|_{H^{s, \tilde{s}}\left(\mathbb{R}_{t} \times \mathbb{R}_{x}^{n}\right)},
$$

where $f=\left(\sigma(X, D) u_{0}\right)^{N}$ and $\tilde{f}=\left(\sigma(X, D) \tilde{u}_{0}\right)^{N}$. Then, on account of (6.3) $)$, we have

$$
\begin{aligned}
& \left\|\langle x\rangle^{-1 / 2+l} \sigma(X, D)(u-\tilde{u})\right\|_{H^{s, \tilde{s}}\left(\mathbb{R}_{t} \times \mathbb{R}_{x}^{n}\right)} \\
& \leq C\left(\sum_{j=0}^{N-1}\left\|\langle x\rangle^{-1 / 2+l} \sigma(X, D) u_{0}\right\|_{H^{s, \tilde{s}}}^{N-1-j} \cdot\left\|\langle x\rangle^{-1 / 2+l} \sigma(X, D) \tilde{u}_{0}\right\|_{H^{s, \tilde{s}}}^{j}\right) \\
& \times\left\|\langle x\rangle^{-1 / 2+l} \sigma(X, D)\left(u_{0}-\tilde{u}_{0}\right)\right\|_{H^{s, \tilde{s}}\left(\mathbb{R}_{t} \times \mathbb{R}_{x}^{n}\right)} .
\end{aligned}
$$

Estimates (6.5) and (6.6) show that, if the first term of the right hand side of (6.5) is sufficiently small, the mapping $u_{0}$ to the solution $u$ for (6.4) is a contraction on the space $X$ which collects all functions $u(t, x)$ with sufficiently small $\|u\|_{X}=$ $\left\|\langle x\rangle^{-1 / 2+l} \sigma(X, D) u\right\|_{H^{s, \tilde{s}}\left(\mathbb{R}_{t} \times \mathbb{R}_{x}^{n}\right)}$. (Note that $\|\cdot\|_{X}$ is not a norm because $\|u\|_{X}=0$ does not always imply $u=0)$. Let us denote this mapping by $\Phi: X \rightarrow X$ noticing that the fixed point of it is a desired time global solution. The contraction here means that we have

$$
\|\Phi(u)-\Phi(\tilde{u})\|_{X} \leq \varepsilon\|u-\tilde{u}\|_{X} \quad(v, \tilde{u} \in X)
$$

with some $0<\varepsilon<1$. Consider the sequence of functions $\left\{u_{n}\right\}_{n \in \mathbb{N}}$ in $X$ defined by $u_{n}=\Phi\left(u_{n-1}\right), u_{0}=0$. Then we have $\left\|u_{n}-u_{m}\right\|_{X} \rightarrow 0$ if $m, n \rightarrow \infty$, which means that $\left\{\langle x\rangle^{-1 / 2+l} \sigma(X, D) u_{n}\right\}_{n \in \mathbb{N}}$ is a Cauchy sequence in $H^{s, \tilde{s}}\left(\mathbb{R}_{t} \times \mathbb{R}_{x}^{n}\right)$. Hence we have a limit

$$
\langle x\rangle^{-1 / 2+l} \sigma(X, D) u_{n} \rightarrow\langle x\rangle^{-1 / 2+l} w
$$

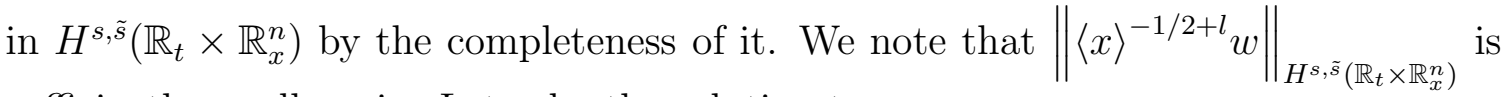
sufficiently small again. Let $u$ be the solution to

$$
\left\{\begin{array}{l}
\left(i \partial_{t}-L\right) u(t, x)=w^{N} \\
u(0, x)=\varphi(x), \quad t \in \mathbb{R}, x \in \mathbb{R}^{n}
\end{array}\right.
$$

We note that $\langle x\rangle^{1 / 2+l} w^{N} \in H^{s, \tilde{s}}\left(\mathbb{R}_{t} \times \mathbb{R}^{n}\right)$ by $(\underline{6.2})$, hence $w^{N} \in C^{0}\left(\mathbb{R}_{t} ; H^{\tilde{s}}\left(\mathbb{R}_{x}^{n}\right)\right)$ by Sobolev's embedding in the variable $t$. Furthermore, on account of the expression

$$
u(t, x)=e^{-i t L} \varphi(x)+\frac{1}{i} \int_{0}^{t} e^{-i(t-s) L} w(s, x)^{N} d s,
$$


we have $u(t, x) \in C^{0}\left(\mathbb{R}_{t} ; H^{\tilde{s}}\left(\mathbb{R}_{x}^{n}\right)\right) \subset C^{0}\left(\mathbb{R}_{t} \times \mathbb{R}_{x}^{n}\right)$ by Sobolev's embedding again. Then, by Corollary 5.5, (6.3), and (6.7), we have

$$
\begin{aligned}
& \left\|\langle x\rangle^{-1 / 2+l} \sigma(X, D)\left(u_{n}-u\right)\right\|_{H^{s, \tilde{s}}\left(\mathbb{R}_{t} \times \mathbb{R}_{x}^{n}\right)} \\
& \leq C\left\|\langle x\rangle^{1 / 2+l}\left(\left(\sigma(X, D) u_{n-1}\right)^{N}-w^{N}\right)\right\|_{H^{s, \tilde{s}}\left(\mathbb{R}_{t} \times \mathbb{R}_{x}^{n}\right)} \rightarrow 0
\end{aligned}
$$

hence we have $w=\sigma(X, D) u$ by (6.7) again. This means that $u$ is the desired time global solution to (6.1) and the proof of Theorem 6.1 is complete.

\section{REFERENCES}

[A] S. Agmon, Spectral properties of Schrödinger operators and scattering theory, Ann. Scuola Norm. Sup. Pisa Cl. Sci. (4) 2 (1975), 151-218.

[BK] M. Ben-Artzi and S. Klainerman, Decay and regularity for the Schrödinger equation, J. Analyse Math. 58 (1992), 25-37.

[Ch] H. Chihara, The initial value problem for cubic semilinear Schrödinger equations, Publ. Res. Inst. Math. Sci. 32 (1996), 445-471.

[Ch2] H. Chihara, Smoothing effects of dispersive pseudodifferential equations, Comm. Partial Differential Equations 27 (2002), 1953-2005.

[HL] G. H. Hardy and J. E. Littlewood, Some Properties of Fractional Integrals, I, Math. Zeit. 27 (1928), 565-606.

[HMN] N. Hayashi, C. Miao and P. I. Naumkin, Global existence of small solutions to the generalized derivative nonlinear Schrodinger equation, Asymptot. Anal. 21 (1999), 133-147.

[HS] I. Herbst, E. Skibsted, Time-dependent approach to radiation conditions, Duke Math. J. 64 (1991), 119-147.

[H] L. Hörmander, The Analysis of Linear Partial Differential Operators II, Springer-Verlag, BerlinNew York, 1983.

[Ho1] T. Hoshiro, On weighted $L^{2}$ estimates of solutions to wave equations, J. Anal. Math. 72 (1997), 127-140.

[Ho2] T. Hoshiro, On the estimates for Helmholtz operator, Tsukuba J. Math. 23 (1999), 131-149.

[I] H. Isozaki, Eikonal equations and spectral representations for long-range Schrödinger Hamiltonians, J. Math. Kyoto Univ. 20 (1980), 243-261.

[K] T. Kato, Wave operators and similarity for some non-selfadjoint operators, Math. Ann. 162 (1966) 258-279.

[KY] T. Kato and K. Yajima, Some examples of smooth operators and the associated smoothing effect, Rev. Math. Phys. 1 (1989), 481-496.

[KM1] S. Klainerman and M. Machedon, Space-time estimates for null forms and the local existence theorem, Comm. Pure Appl. Math. 46 (1993), 1221-1268.

[KM2] S. Klainerman and M. Machedon, Smoothing estimates for null forms and applications, Duke Math. J. 81 (1995), 99-133.

[Ku] H. Kumano-go, Pseudodifferential operators, MIT Press, Cambridge, Mass.-London, 1981.

[KW] D. S. Kurtz and R. L. Wheeden, Results on weighted norm inequalities for multipliers, Trans. Amer. Math. Soc. 255 (1979), 343-362.

[OZ] T. Ozawa and J. Zhai, Global existence of small classical solutions to nonlinear Schrödinger equations, Ann. Inst. H. Poincaré Anal. Non Linéaire 25 (2008), 303-311.

[ReS] M. Reed and B. Simon, Methods of modern mathematical physics. III. Scattering theory, Academic Press, New York-London, 1979

[RS1] M. Ruzhansky and M. Sugimoto, Global $L^{2}$-boundedness theorems for a class of Fourier integral operators, Comm. Partial Differential Equations, 31 (2006), 547-569.

[RS2] M. Ruzhansky and M. Sugimoto, A smoothing property of Schrödinger equations in the critical case, Math. Ann. 335 (2006), 645-673. 
[RS3] M. Ruzhansky and M. Sugimoto, A smoothing property of Schrödinger equations and a global existence result to derivative nonlinear equations, Advances in analysis, 315-320, World Sci. Publ., Hackensack, NJ, 2005.

[SW] E. M. Stein and G. Weiss, Fractional integrals on n-dimensional Euclidean space, J. Math. Mech. 7 (1958), 503-514.

[Su1] M. Sugimoto, Global smoothing properties of generalized Schrödinger equations, J. Anal. Math. 76 (1998), 191-204.

[Su2] M. Sugimoto, A Smoothing property of Schrödinger equations along the sphere, J. Anal. Math. 89 (2003), 15-30.

[SuT] M. Sugimoto and K. Tsujimoto, A resolvent estimate and a smoothing property of inhomogeneous Schrödinger equations, Proc. Japan Acad. Ser. A Math. Sci. 74 (1998), 74-76.

[W] K. Watanabe, Smooth perturbations of the selfadjoint operator $|\Delta|^{\alpha / 2}$, Tokyo J. Math. 14 (1991), 239-250.

Michael RuZHANSKY:

Department of Mathematics

IMPERIAL COLLEGE LONDON

180 QueEn's Gate, London SW7 2AZ, UK

E-mail address m.ruzhansky@imperial.ac.uk

Mitsuru Sugimoto:

Graduate School of Mathematics

NAGOYA UNIVERSITY

Furocho, Chikusa-Ku, Nagoya 464-8602, Japan

E-mail address sugimoto@math.nagoya-u.ac.jp 